\title{
Worst Case Analysis of Age of Information in a Shared-Access Channel
}

\author{
Mohammad Moltafet and Markus Leinonen \\ Centre for Wireless Communications - Radio Technologies \\ University of Oulu, Finland \\ e-mail: \{mohammad.moltafet, markus.leinonen\}@oulu.fi
}

\author{
Marian Codreanu \\ Department of Science and Technology \\ Linköping University, Sweden \\ e-mail: marian.codreanu@liu.se
}

\begin{abstract}
Freshness of status update packets is essential for enabling a wide range of Internet of Things (IoT) applications. In this paper, we consider a status update system in which various sensors are assigned to transmit status update packets of a physical process to a desired destination. We consider that the sensors share a wireless channel and contend for the channel access based on a carrier sense multiple access (CSMA) method. We study freshness of the status update system at the destination using the age of information (AoI) metric. To this end, we analyze the worst case average AoI for each sensor in the CSMA-based system. Numerical results show that the AoI in the CSMA-based system may dramatically increase when the number of sensors increases. Moreover, we observe that the contention window size and the packet arrival rate must be optimized since they have a critical role in the performance of the system.

Index Terms- Age of information (AoI), multi-access channel, CSMA/CA, M/G/1 queueing model.
\end{abstract}

\section{INTRODUCTION}

In a wide range of Internet of Things (IoT) applications, such as surveillance in smart home systems and drone control, the destination requires the status information of a few physical processes collected from various sensors. In these applications, timeliness of the status information is very critical. The age of information (AoI) was introduced as a destination centric metric that characterizes this timeliness [1]. The AoI for a sensor is defined as the time elapsed since the last received status update packet was generated at the sensor. To evaluate the AoI, the most commonly used metric is average AoI [2].

Due to the scarcity of spectrum in wireless networks, and simplicity of sensors, it is critical to exploit an appropriate access protocol that allows different sensors to efficiently use a shared channel to send their status update packets. Carrier sense multiple access with collision avoidance (CSMA/CA) is the most simple and practical access technique in the wireless networks. CSMA/CA is a distributed access scheme that allows each sensor to start the transmission process without any admission whenever it has a data packet to transmit. In this access scheme, the different nodes contend with each other to access the shared channel. Two versions of CSMA/CA are employed: I) basic CSMA/CA, and II) CSMA/CA with channel reservation. The basic CSMA/CA technique is appropriate for the systems with short data packets and the systems where the hidden node problem is negligible [3]. The CSMA/CA technique with channel reservation is appropriate for the systems with long data packets. Moreover, it solves the hidden node problem of CSMA/CA-based systems by exploiting the request-to-send/clear-to-send (RTS/CTS) mechanism. There are many works that have evaluated the performance of a CSMA/CA-based system and optimized the system with respect to different criteria. However, there are only a few works that have studied the freshness in a CSMA/CA-based system.

The authors of [4] using simulation investigated the AoI in a CSMA/CA-based vehicular network. The authors of [5] investigated ALOHA and scheduled based access techniques in wireless sensor networks and minimized the average AoI by optimizing the probability of transmission in each node. The most related work to our work is [6]. The authors of this work analyzed the AoI in a CSMA/CA-based system using the stochastic hybrid systems technique. They assumed that the status update packets of each node are generated according to the Poisson process, the queue size of each node is zero, and the total capacity of the queuing system is one packet under service. In addition, they considered that a packet being served is replaced if a new packet arrives. They optimized the average AoI by calibrating the back-off time of each link.

In this paper, we analytically calculate the worst case average AoI of each sensor in a simplified CSMA/CA-based system with the first-come first-served (FCFS) policy and infinite queue size. As presented above, the existing works have not analytically evaluated the AoI in such a network model. The worst case analysis is carried out by considering that when a sensor contends for the channel to transmit its status update packet, all the other sensors have a packet to transmit. Therefore, we consider a saturated system where the probability of collisions in the system has the highest value. We confine to the worst case analysis because a more general case in which the other sensors do not have saturated queues is intractable to analyze.

The rest of this paper is organized as follows. Section II presents the system model and the AoI metric. In Section III, the average AoI of the simplified CSMA/CA-based system is calculated. Numerical results are presented in Section IV and conclusions are drawn in Section V. 


\section{System Model AND AoI Metrics}

We consider a simplified CSMA/CA-based wireless sensor network consisting of $M$ sensors denoted by $\mathcal{M}=$ $\{1, \ldots, M\}$. Each sensor is assigned to send status update packets of a random process to a desired destination. In this paper, we study the AoI of one sensor, $m$, in a worst case scenario where all the other sensors $m^{\prime} \in \mathcal{M} \backslash\{m\}$ always have a packet to transmit, i.e., they have saturated queues. In this scenario, the probability of collisions for sensor $m$ has the highest value. We assume that the packet arrival rate of sensor $m$ follows the Poisson process with rate $\lambda$, and the server of sensor $m$ works according to the FCFS policy. From the view point of sensor $m$, we in general have an M/G/1 queuing model in which the service time parameters are determined according to the CSMA/CA technique. In the following, a simplified CSMA/CA technique and the metrics to evaluate the AoI are presented.

1) CSMA/CA Mechanism: Here, we briefly present the main concept of the basic CSMA/CA technique standardized by IEEE 802.11 . When sensor $m$ has a packet to transmit, it monitors the shared channel. If the channel is idle for a predetermined period time named distributed interframe space (DIFS), the sensor transmits. Otherwise, if the channel is sensed busy, the sensor persists to monitor the channel until it is found idle for a DIFS period. At this point, the sensor generates a random number $w$ according to the discrete uniform distribution taking values in $\{1, \ldots, W\}$, where $W$ represents the number of slots in a contention window. We assume a fixed $W$. This random waiting time is called back-off time and brings the collision avoidance feature of the protocol. After choosing the random number, there is a back-off time counter in the sensor that is decremented as long as the shared channel is sensed idle. When a transmission is detected, the counter is frozen. When the channel is sensed idle for DIFS, the counter is reactivated. The sensor starts to transmit its data when the counter reaches zero. After transmitting the data, the transmitter senses the channel to detect the acknowledgment (ACK) message from the destination. If the transmitter does not receive ACK within a predetermined time called $T_{\mathrm{ACK}}$, or it detects a signal of other sensors in the channel, it reschedules the packet transmission according to the random back-off rule. After a successful transmission, if the sensor has a packet in its buffer waiting to be transmitted, the process of transmission is started by the random back-off rule.

2) AoI: A status update packet of each sensor contains the measured value of the monitored process and a time stamp that represents the time when the sample was generated. If at a time instant $t$, the most recently received status update packet contains the time stamp $U(t)$, AoI is defined as the random process $\Delta(t)=t-U(t)$. In other words, the AoI measures for each sensor the time elapsed since the last received status update packet was generated at the sensor. The most common metric of the AoI is average AoI. The average AoI of a sensor is the average of $\Delta(t)$. In the following, the average AoI expression of an $\mathrm{M} / \mathrm{G} / 1$ queueing model is presented.
3) Average AoI for an M/G/l Queueing Model: Let $S$ denote the service time, $\Delta$ denote the average AoI of an M/G/1 queueing model. Thus, we have [7]

$$
\Delta=\mathbb{E}[S]+\frac{\lambda \mathbb{E}\left[S^{2}\right]}{2(1-\lambda \mathbb{E}[S])}+\frac{1-\lambda \mathbb{E}[S]}{\lambda L_{S}(\lambda)},
$$

where $\mathbb{E}[S]$ is the expectation of the service time, $\mathbb{E}\left[S^{2}\right]$ is the second moment of the service time, and $L_{S}(\lambda)$ is the Laplace transform of the probability distribution function of service time at the packet arrival rate $\lambda$.

\section{Average AoI of the CSMA/CA-BASEd System}

In general, the considered system model for sensor $m$ is identical to an M/G/1 queueing model where the service time parameters are determined by a basic CSMA/CA-based system. However, calculating the service time parameters of a CSMA/CA/based system is intractable due to the intricate nature of the contention mechanism which results in a dependency of the transmissions of the different sensors. In this regard, to characterize the probability of a successful transmission in each attempt, we use the approximations in [3] as follows. We assume that the probability of a collision in each slot for each sensor has a fixed value which disregards the dependencies of the transmission states of other sensors as well as the number of retransmissions. Moreover, we assume that a packet transmission process is started by the random back-off rule and that the ACK message is instantaneous and error-free.

To calculate the average AoI in (1), we need to determine the expectation of the service time $\mathbb{E}[S]$, the second moment of the service time $\mathbb{E}\left[S^{2}\right]$, and the Laplace transform of the service time $L_{S}(\lambda)$. To this end, we characterize the required time for each successful and unsuccessful transmission in a channel access attempt. Let a random variable $\xi_{j}$ denote the elapsed time of successful transmission of sensor $m$ at the $j$ th attempt. Based on the value of number $w, \xi_{j}$ is defined as

$$
\xi_{j}(w)=\sum_{i=1}^{w} T_{i}+T_{\mathrm{S}}
$$

where $T_{i}$ is the time interval between back-off time counters $i$ and $i-1$ which in general varies over time; $T_{\mathrm{S}}$ is the required time for a successful packet transmission which is given by $T_{\mathrm{S}}=T_{\mathrm{P}}$, where $T_{\mathrm{P}}$ is the required time to transmit a data packet (which is determined according to the channel rate and data packet size). For $T_{i}$, there are two possibilities, which are determined based on the activities of other $M-1$ sensors $m^{\prime} \in \mathcal{M} \backslash\{m\}$ as follows. I) When there is a successful or unsuccessful transmission of one of the other sensors between back-off time counters $i$ and $i-1, T_{i}$ is equal to $T_{i}=T_{\mathrm{P}}+T_{\mathrm{DIFS}}$, where $T_{\mathrm{DIFS}}$ is the DIFS time. II) When there is no transmission by the other sensors $m^{\prime} \in \mathcal{M} \backslash\{m\}$ between back-off time counters $i$ and $i-1, T_{i}$ is equal to $T_{i}=T_{\mathrm{F}}$, where $T_{\mathrm{F}}$ is the maximum duration that the sensor persists to sense the channel before decrementing the counter. 
In addition, we introduce a random variable $\zeta_{j}$ that represents the time that sensor $m$ spends when the $j$ th attempt is unsuccessful. Based on the value of number $w, \zeta_{j}$ is given by

$$
\zeta_{j}(w)=\sum_{i=1}^{w} T_{i}+T_{\mathrm{C}}
$$

where $T_{\mathrm{C}}$ is the elapsed time for the unsuccessful packet transmission which is given by $T_{\mathrm{C}}=T_{\mathrm{P}}$. From here onwards, for simplicity in notation, we use $\xi_{j}$ and $\zeta_{j}$ instead of $\xi_{j}(w)$ and $\zeta_{j}(w)$, respectively.

Consider that sensor $m$ successfully transmits its data at the $K^{\text {th }}$ attempt. Using (2) and (3), the service time is a random variable

$$
S_{K}=\sum_{j=1}^{K-1} \zeta_{j}+\xi_{K}
$$

Equation (4) expresses that $K-1$ attempts are failed and the $K^{\text {th }}$ attempt is successful. Let $P_{\mathrm{S}}$ denote the probability of a successful transmission for sensor $m$ in each contention interval. Then, the expectation, second moment, and Laplace transform of the service time, denoted by $\mathbb{E}[S], \mathbb{E}\left[S^{2}\right]$, and $\mathbb{E}\left[e^{-\lambda S}\right]$, are calculated as follows:

$$
\begin{aligned}
& \mathbb{E}[S]=\mathbb{E}\left[\mathbb{E}\left[S_{K} \mid K\right]\right] \\
& =\sum_{k=1}^{\infty} \mathbb{E}\left[S_{K} \mid K=k\right] P_{\mathrm{S}}\left(1-P_{\mathrm{S}}\right)^{k-1}, \\
& \mathbb{E}\left[S^{2}\right]=\mathbb{E}\left[\mathbb{E}\left[S_{K}^{2} \mid K\right]\right] \\
& =\sum_{k=1}^{\infty} \mathbb{E}\left[S_{K}^{2} \mid K=k\right] P_{\mathrm{S}}\left(1-P_{\mathrm{S}}\right)^{k-1}, \\
& \mathbb{E}\left[e^{-\lambda S}\right]=\mathbb{E}\left[\mathbb{E}\left[e^{-\lambda S_{K}} \mid K\right]\right] \\
& =\sum_{k=1}^{\infty} \mathbb{E}\left[e^{-\lambda S_{K}} \mid K=k\right] P_{\mathrm{S}}\left(1-P_{\mathrm{S}}\right)^{k-1},
\end{aligned}
$$

where term $P_{\mathrm{S}}\left(1-P_{\mathrm{S}}\right)^{k-1}$ indicates that $k-1$ attempts are failed and the $k^{\text {th }}$ attempt is successful. To calculate $P_{\mathrm{S}}$, we use the results in [3]. Therefore, we have

$$
P_{\mathrm{S}}=\left(\frac{W-1}{W+1}\right)^{M-1} \text {. }
$$

It is worth to note that (6) relies on the approximations we made to simplify the analysis.

By using (4), $\mathbb{E}\left[S_{K} \mid K=k\right], \quad \mathbb{E}\left[S_{K}^{2} \mid K=k\right], \quad$ and $\mathbb{E}\left[e^{-\lambda S_{K}} \mid K=k\right]$ in (5) are calculated as follows:

$$
\begin{aligned}
& \mathbb{E}\left[S_{K} \mid K=k\right]=\sum_{j=1}^{k-1} \mathbb{E}\left[\zeta_{j}\right]+\mathbb{E}\left[\xi_{k}\right], \\
& \mathbb{E}\left[S_{K}^{2} \mid K=k\right]=\mathbb{E}\left[\left(\sum_{j=1}^{k-1} \zeta_{j}+\xi_{k}\right)^{2}\right]=\mathbb{E}\left[\sum_{j=1}^{k-1} \zeta_{j}^{2}+\right. \\
& \left.2 \sum_{j=1}^{k-1} \sum_{j^{\prime}=1, j^{\prime} \neq j}^{k-1} \zeta_{j} \zeta_{j^{\prime}}+\xi_{k}^{2}+2 \xi_{k} \sum_{j=1}^{k-1} \zeta_{j}\right]
\end{aligned}
$$

$$
\mathbb{E}\left[e^{-\lambda S_{K}} \mid K=k\right]=\prod_{j=1}^{k-1} \mathbb{E}\left[e^{-\lambda \zeta_{j}}\right] \mathbb{E}\left[e^{-\lambda \xi_{k}}\right] .
$$

Since the service time of each channel access attempt is independent of the service time of other attempts, we have

$$
\begin{aligned}
& \mathbb{E}\left[\zeta_{j} \zeta_{j^{\prime}}\right]=\mathbb{E}\left[\zeta_{j}\right] \mathbb{E}\left[\zeta_{j^{\prime}}\right], \forall j, j^{\prime}, \quad j \neq j^{\prime}, \\
& \mathbb{E}\left[\zeta_{j} \xi_{j^{\prime}}\right]=\mathbb{E}\left[\zeta_{j}\right] \mathbb{E}\left[\xi_{j^{\prime}}\right], \forall j, j^{\prime}, \quad j \neq j^{\prime}, \\
& \mathbb{E}\left[e^{-\lambda \zeta_{j}} e^{-\lambda \zeta_{j^{\prime}}}\right]=\mathbb{E}\left[e^{-\lambda \zeta_{j}}\right] \mathbb{E}\left[e^{-\lambda \zeta_{j^{\prime}}}\right], \forall j, j^{\prime}, \quad j \neq j^{\prime}, \\
& \mathbb{E}\left[e^{-\lambda \zeta_{j}} e^{-\lambda \xi_{j^{\prime}}}\right]=\mathbb{E}\left[e^{-\lambda \zeta_{j}}\right] \mathbb{E}\left[e^{-\lambda \xi_{j^{\prime}}}\right], \forall j, j^{\prime}, \quad j \neq j^{\prime} .
\end{aligned}
$$

Moreover, since the elapsed time of each successful (unsuccessful) transmission for different attempts have a same distribution, we have

$$
\begin{aligned}
& \mathbb{E}\left[\zeta_{j}\right]=\mathbb{E}\left[\zeta_{j^{\prime}}\right]=\bar{\zeta}_{1}, \mathbb{E}\left[\zeta_{j}^{2}\right]=\mathbb{E}\left[\zeta_{j^{\prime}}^{2}\right]=\bar{\zeta}_{2}, \\
& \mathbb{E}\left[e^{-\lambda \zeta_{j}}\right]=\mathbb{E}\left[e^{-\lambda \zeta_{j^{\prime}}}\right]=\bar{\zeta}_{3}, \forall j, j^{\prime}, j \neq j^{\prime}, \\
& \mathbb{E}\left[\xi_{j}\right]=\mathbb{E}\left[\xi_{j^{\prime}}\right]=\bar{\xi}_{1}, \mathbb{E}\left[\xi_{j}^{2}\right]=\mathbb{E}\left[\xi_{j^{\prime}}^{2}\right]=\bar{\xi}_{2}, \\
& \mathbb{E}\left[e^{-\lambda \xi_{j}}\right]=\mathbb{E}\left[e^{-\lambda \xi_{j^{\prime}}}\right]=\bar{\xi}_{3}, \forall j, j^{\prime}, j \neq j^{\prime} .
\end{aligned}
$$

Using (8) and (9), (7) is written as

$$
\begin{aligned}
& \mathbb{E}\left[S_{K} \mid K=k\right]=(k-1) \bar{\zeta}_{1}+\bar{\xi}_{1}, \\
& \mathbb{E}\left[S_{K}^{2} \mid K=k\right]=(k-1) \bar{\zeta}_{2}+(k-1)(k-2) \bar{\zeta}_{1}^{2} \\
& +\bar{\xi}_{2}+2(k-1) \bar{\zeta}_{1} \bar{\xi}_{1}, \\
& \mathbb{E}\left[e^{-\lambda S_{K}} \mid K=k\right]=\bar{\zeta}_{3}^{k-1} \bar{\xi}_{3} .
\end{aligned}
$$

Combining (5) and (10), we have

$$
\begin{aligned}
& \mathbb{E}[S]=\sum_{k=1}^{\infty}\left((k-1) \bar{\zeta}_{1}+\bar{\xi}_{1}\right) P_{\mathrm{S}}\left(1-P_{\mathrm{S}}\right)^{k-1}, \\
& \mathbb{E}\left[S^{2}\right]=\sum_{k=1}^{\infty}\left((k-1) \bar{\zeta}_{2}+(k-1)(k-2) \bar{\zeta}_{1}^{2}+\bar{\xi}_{2}\right. \\
& \left.+2(k-1) \bar{\zeta}_{1} \bar{\xi}_{1}\right) P_{\mathrm{S}}\left(1-P_{\mathrm{S}}\right)^{k-1}, \\
& \mathbb{E}\left[e^{-\lambda S}\right]=\sum_{k=1}^{\infty} \bar{\zeta}_{3}^{k-1} \bar{\xi}_{3} P_{\mathrm{S}}\left(1-P_{\mathrm{S}}\right)^{k-1} .
\end{aligned}
$$

According to the feature of the series, for each $0 \leq \alpha<1$ we have $[8$, Sect. 8.6]

$$
\begin{aligned}
\sum_{k=1}^{\infty} k \alpha^{k} & =\frac{\alpha}{(1-\alpha)^{2}}, \\
\sum_{k=1}^{\infty} k^{2} \alpha^{k} & =\frac{\alpha(1+\alpha)}{(1-\alpha)^{3}} .
\end{aligned}
$$

Finally, by applying (12) in (11), $\mathbb{E}[S], \mathbb{E}\left[S^{2}\right]$, and $\mathbb{E}\left[e^{-\lambda S}\right]$ are calculated as follows:

$$
\begin{aligned}
& \mathbb{E}[S]=\bar{\xi}_{1}-\bar{\zeta}_{1}+\frac{\bar{\zeta}_{1}}{P_{\mathrm{S}}} \\
& \mathbb{E}\left[S^{2}\right]=2 \bar{\zeta}_{1}^{2}+\bar{\xi}_{2}-\bar{\zeta}_{2}-2 \bar{\zeta}_{1} \bar{\xi}_{1}+\frac{2 \bar{\zeta}_{1} \bar{\xi}_{1}+\bar{\zeta}_{2}-3 \bar{\zeta}_{1}^{2}}{P_{\mathrm{S}}} \\
& +\frac{\bar{\zeta}_{1}^{2}\left(2-P_{\mathrm{S}}\right)}{P_{\mathrm{S}}^{2}} .
\end{aligned}
$$




$$
\mathbb{E}\left[e^{-\lambda S}\right]= \begin{cases}\frac{\bar{\xi}_{3} P_{\mathrm{S}}}{1-\bar{\zeta}_{3}+\bar{\zeta}_{3} P_{\mathrm{S}}}, & \bar{\zeta}_{3}\left(1-P_{\mathrm{S}}\right)<1, \\ \infty, & \text { Otherwise. }\end{cases}
$$

In the following, we present the detailed steps of calculating $\bar{\xi}_{1}$, the other parameters $\bar{\xi}_{2}, \bar{\xi}_{3}, \bar{\zeta}_{1}, \bar{\zeta}_{2}$, and $\bar{\zeta}_{3}$ can easily be derived by using the same steps. $\bar{\xi}_{1}$ can be written as follows:

$$
\bar{\xi}_{1}=\sum_{l=1}^{W} \mathbb{E}\left[\xi_{j}(w) \mid w=l\right] P(w=l),
$$

where due to the uniform distribution of random backoff selection we have $P(w=l)=1 / W$, and by using (2), $\mathbb{E}\left[\xi_{j}(w) \mid w=l\right]$ is written as follows:

$$
\begin{aligned}
\mathbb{E}\left[\xi_{j}(w) \mid w=l\right] & =\mathbb{E}\left[\sum_{i=1}^{l} T_{i}+T_{\mathrm{S}}\right] \\
& \stackrel{(a)}{=} \mathbb{E}\left[\sum_{i=1}^{l} T_{i}\right]+T_{\mathrm{S}} \stackrel{(b)}{=} l \mathbb{E}[T]+T_{\mathrm{S}},
\end{aligned}
$$

where equality (a) follows from the fact that $T_{\mathrm{S}}$ is a fixed time interval; equality (b) comes from the fact that the time between each two consecutive back-off time counters $i$ and $i-1$ has a same distribution, and thus, we have $\mathbb{E}\left[T_{1}\right]=\cdots=\mathbb{E}\left[T_{l}\right]=\mathbb{E}[T]$. From the definition of $T_{i}$ presented after (2), we have

$$
\mathbb{E}[T]=\left(1-P_{\mathrm{tr}}\right) T_{\mathrm{F}}+P_{\mathrm{tr}}\left(T_{\mathrm{P}}+T_{\mathrm{DIFS}}\right),
$$

where $P_{\text {tr }}$ is the probability of having at least one transmission in each slot when the other sensors $m^{\prime} \in \mathcal{M} \backslash\{m\}$ are contending to access the channel which is given as [3]

$$
P_{\mathrm{tr}}=1-\left(\frac{W-1}{W+1}\right)^{M-1} \text {. }
$$

\section{NUMERICAL RESUlTS}

In this section, we present numerical results to show the performance of the system in terms of the AoI versus different system parameters. The considered parameters for numerical results are: $T_{\mathrm{DIFS}}=128 \mu \mathrm{s}$, channel bit rate $1 \mathrm{Mbit} / \mathrm{s}$, and packet size 300 Bytes.

Figs. 1 depicts the average AoI of sensor $m$ as a function of $\lambda$ for different number of sensors with a fixed contention window size. As it can be seen from the curvatures, by increasing the number of sensors, the average AoI dramatically increases because the probability of collisions in the system increases. In addition, when the number of sensors increases, the value of the packet arrival rate $\lambda$ that minimizes the average AoI decreases.

Fig. 2 illustrates the average AoI of sensor $m$ as a function of $\lambda$ for different contention window sizes with a fixed number of sensors $M=110$. According to this figure, naively increasing (or decreasing) the contention window size does not minimize the average AoI. Namely, $W=500$ leads to a smaller average AoI than both $W=100$ and $W=1000$.

In addition, we can conclude from the figures that generating the status update packets neither too frequently nor too rarely minimizes the average AoI.

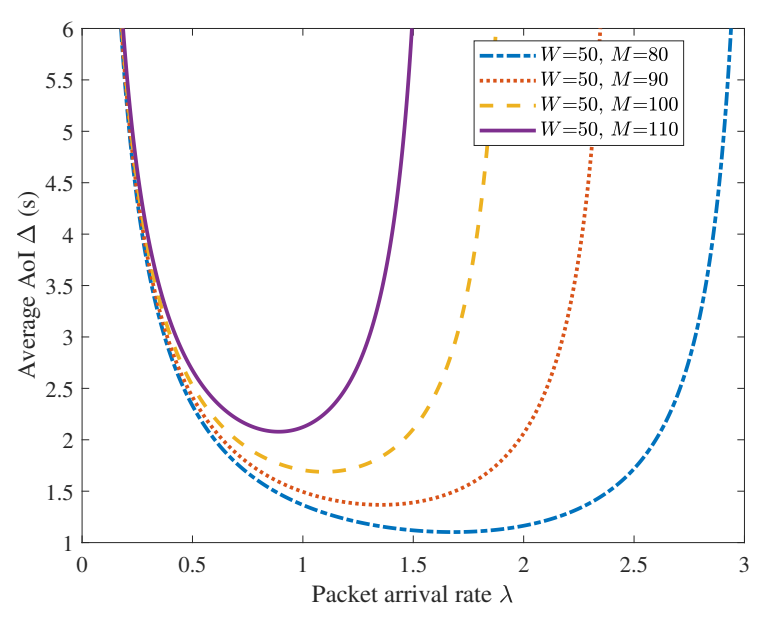

Fig. 1: The average AoI of sensor $m$ as a function of $\lambda$ for different number of sensors with a fixed contention window size $W=50$.

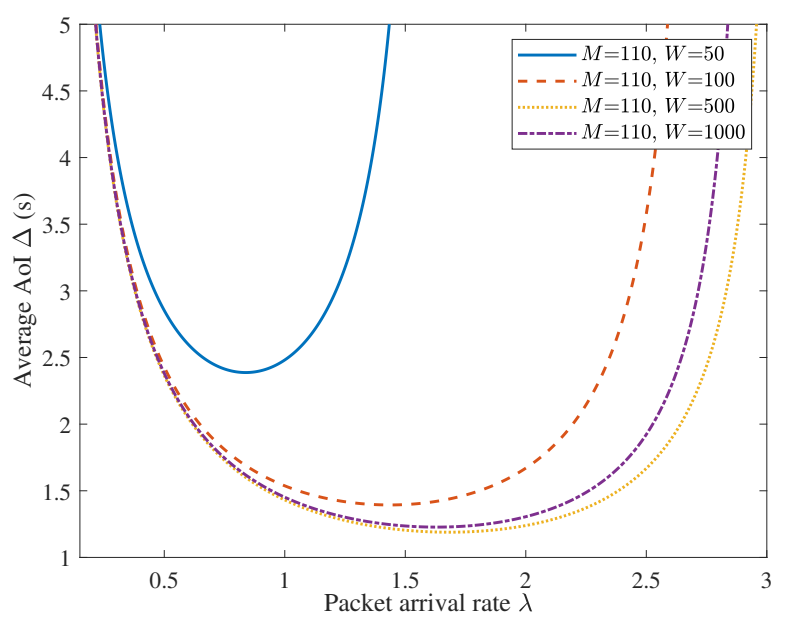

Fig. 2: The average AoI of sensor $m$ as a function of $\lambda$ for different contention window sizes with a fixed number of sensors $M=110$.

\section{CONCLusion}

In this paper, we analytically evaluated the worst case average AoI of a simplified CSMA/CA-based system. The worst case analysis was carried out by considering a scenario in which the probability of collisions for the considered sensor has the highest value. According to the numerical results, the number of contending sensors significantly affects the AoI of a sensor due to network congestion. In addition, the experiments illustrated that if one optimizes the contention window size and packet arrival rate, significant improvements in the freshness of status updates can be achieved in the considered system.

\section{ACKNOWLEDGEMENT}

This research has been financially supported by Infotech Oulu. M. Codreanu would like to acknowledge the support of the European Union's Horizon 2020 research and innovation programme under the Marie Skłodowska-Curie Grant 
Agreement No. 793402 (COMPRESS NETS). This research has also been financially supported by Academy of Finland 6Genesis Flagship (grant 318927) and Finnish Foundation for Technology Promotion.

\section{REFERENCES}

[1] S. Kaul, R. Yates, and M. Gruteser, "Real-time status: How often should one update?" in Proc. IEEE Int. Conf. on Computer. Commun. (INFOCOM), Orlando, FL, USA, Mar. 25-30, 2012, pp. 2731-2735.

[2] A. Kosta, N. Pappas, and V. Angelakis, "Age of information: A new concept, metric, and tool," Foun. and Trends in Net., vol. 12, no. 3, pp. 162-259, 2017.

[3] G. Bianchi, "Performance analysis of the IEEE 802.11 distributed coordination function," IEEE Trans. Appl. Superconduct., vol. 18, no. 3, pp. 535-547, Mar. 2000.

[4] S. Kaul, M. Gruteser, V. Rai, and J. Kenney, "Minimizing age of information in vehicular networks," in Proc. Commun. Society. Conf. on Sensor, Mesh and Ad Hoc Commun. and Net., Salt Lake City, UT, USA, Jun. 27-30, 2011, pp. 350-358.

[5] R. D. Yates and S. K. Kaul, "Status updates over unreliable multiaccess channels," in Proc. IEEE Int. Symp. Inform. Theory, Aachen, Germany, Jun. 25-30, 2017, pp. 331-335.

[6] A. Maatouk, M. Assaad, and A. Ephremides, "Minimizing the age of information in a CSMA environment," [Online]. Available: https://arxiv.org/abs/1901.00481, 2019.

[7] Y. Inoue, H. Masuyama, T. Takine, and T. Tanaka, "The stationary distribution of the age of information in FCFS single-server queues," in Proc. IEEE Int. Symp. Inform. Theory, Aachen, Germany, Jun. 25-30, 2017, pp. 571-575.

[8] L. Rade and B. Westergren, Mathematics Handbook for Science and Engineering. Berlin, Germany: Springer, 2005. 